\title{
Fast off-rate CD229 chimeric antigen receptor T cells efficiently target multiple myeloma, spare T cells, and exhibit reduced trogocytosis
}

Erica R. Vander Mause ${ }^{1,2,3}$, Jillian M. Baker ${ }^{1}$, Sabarinath V. Radhakrishnan ${ }^{3,4}$, Patricia Davis ${ }^{5,6}$, Jens Panse ${ }^{7}$, James E. Marvin ${ }^{8}$, Michael L. Olson ${ }^{6}$, Mary Steinbach², David P. Ng${ }^{6}$, Carol S. Lim³ ${ }^{3}$ Djordje Atanackovic ${ }^{1,9}$, Tim Luetkens ${ }^{1,2,9^{*}}$

${ }^{1}$ Department of Microbiology and Immunology, University of Maryland School of Medicine, Baltimore, MD, United States

${ }^{2}$ Hematology and Hematologic Malignancies, Huntsman Cancer Institute, University of Utah, Salt Lake City, UT, United States

${ }^{3}$ Department of Pharmaceutics \& Pharmaceutical Chemistry, University of Utah, Salt Lake City, UT, United States

${ }^{4}$ Division of Hematology and Oncology, Medical College of Wisconsin, Milwaukee, USA ${ }^{5}$ Division of Experimental and Clinical Pathology, ARUP Laboratories, Salt Lake City, USA

${ }^{6}$ Department of Pathology, University of Utah, Salt Lake City, UT, United States

${ }^{7}$ Department of Oncology, Hematology, Hemostaseology, and Stem Cell

Transplantation, University Hospital RWTH Aachen, Aachen, Germany

${ }^{8}$ Huntsman Cancer Institute, University of Utah, Salt Lake City, UT, United States

${ }^{9}$ Department of Medicine and Transplant/Cell Therapy Program, University of Maryland School of Medicine and Marlene and Stewart Greenebaum Comprehensive Cancer Center, Baltimore, MD, United States

One sentence summary: Affinity tuning approach yields low affinity CD229 CAR binding domain maintaining the parental clone's anti-tumor activity while eliminating killing of healthy T cells, increasing CAR T cell expansion, and decreasing trogocytosis.

Word count (abstract): 175

Word count (main text): 2,535

${ }^{*}$ Corresponding author:

Tim Luetkens, MD

Assistant Professor

Director R\&D at Fannie Angelos Cellular Therapeutics GMP Laboratory

Department of Microbiology and Immunology

University of Maryland

655 W Baltimore St, RM. 9-009

Baltimore, MD 21201

Phone: 410-706-1924

Email: tluetkens@som.umaryland.edu 


\section{ABSTRACT}

T cells expressing chimeric antigen receptors have shown remarkable therapeutic activity against different types of cancer. However, their wider use has been hampered by the potential for life-threatening toxicities due to the unintended targeting of healthy cells expressing low levels of the targeted antigen. We have now developed an affinity-tuning approach for the generation of minimally modified, low-affinity antibody variants derived from existing highaffinity antibodies. Using this approach, we engineered low affinity variants of the fully human CD229-specific antibody 2D3. Parental 2D3 originally efficiently targeted multiple myeloma cells but also healthy T cells expressing low levels of CD229. We demonstrate that CAR T cells based on a low affinity variant of 2D3 maintain the parental antibody's anti-tumor activity, but lack its targeting of healthy T cells. In addition, variant CD229 CAR T cells show reduced trogocytosis potentially augmenting CAR T cell persistence. The fast off-rate CAR produced using our affinity tuning approach eliminates a key liability of CD229 CAR T cells and paves the way for the effective and safe treatment of patients with multiple myeloma. 


\section{MAIN TEXT}

T cells expressing chimeric antigen receptors using antibodies to target cancer-associated surface antigens have been shown to be highly effective against several hematologic malignancies, including B cell lymphoma ${ }^{1}$ and multiple myeloma ${ }^{2,3}$. However, their extraordinary cytotoxic activity poses new challenges, such as the unintended killing of healthy tissues expressing the targeted antigen often at substantially lower levels ${ }^{4}$. In the case of the widely used CD19 CAR T cells, this on-target off-tumor toxicity results in the elimination of healthy B cells ${ }^{5,6}$ and various other CAR T cell approaches have resulted in life-threatening toxicities and even patient deaths due to the targeting of healthy tissues ${ }^{7-9}$. It has been shown previously that CAR T cells exert potent anti-tumor activity across a wide range of affinities ${ }^{10-12}$ and many CAR T cell strategies currently in clinical use likely exceed the required affinity threshold. As a consequence, several low affinity antibodies have been developed for a number of cancer targets to increase cancer selectivity as well as CAR T cell persistence and function ${ }^{13-}$ ${ }^{16}$. However, none of these clones were derived from the extensively tested high affinity antibodies already in clinical use and had to again undergo rigorous preclinical evaluation with the risk for substantial liabilities, such as off-target reactivity, unstable epitopes, and tonic signaling.

We have now developed an affinity-tuning platform for the generation of low-affinity antibody variants derived from existing high-affinity antibodies. We demonstrate that CAR T cells based on antibody variants developed using this approach show increased selectivity for tumor cells, increased expansion, maintained anti-tumor activity in vitro, and reduced trogocytosis, the stripping of target antigen from tumor cells by CAR T cells, potentially augmenting their 
persistence in vivo. We hypothesize that approaches enabling the systematic reduction in antibody affinity of existing CAR binding domains will become an important tool in the development of more effective CAR T cell approaches.

\section{RESULTS}

Generation of CD229 antibody variants to increase CAR T cell selectivity

CAR T cells targeting BCMA, an antigen otherwise exclusively expressed on plasma cells, have been approved for the treatment of multiple myeloma $(\mathrm{MM})^{17,18}$. However, most patients relapse within the first year ${ }^{2}$ potentially due to incomplete targeting of MM-propagating cells in the memory B cell pool ${ }^{19,20}$. Recently, an alternative CAR T cell approach based on the phagedisplay derived, fully human anti-CD229 antibody 2D3 was developed showing targeting not only of terminally differentiated MM plasma cells but also of MM-propagating cells ${ }^{21}$. While CD229 CAR T cells indeed show efficient targeting of MM cells (Fig. 1A, Suppl. Fig. 1), they also target healthy T cells (Fig. 1B), indicating the potential for substantial toxicities. Analyzing expression of CD229 on MM cells and normal T cells from patients with relapsed-refractory MM using flow cytometry, we found that T cells express significantly lower levels of CD229 than MM cells (Fig. 1C).

Affinity tuning of CAR binding domains has previously been shown to reduce targeting of cells expressing lower levels of the targeted antigen (Fig. 1D). Compared to other commonly used CAR binding domains, such as the CD19-specific antibody FMC6322, the affinity of wildtype 2D3 is already relatively low at $476 \mathrm{nM}$ (Fig. 1E). Considering the high specificity and extensive preclinical characterization of 2D3, as well as the established anti-tumor activity and functionality of 2D3-based CAR T cells ${ }^{21}$, we developed an affinity tuning platform to generate 
low affinity variants of 2D3 comprehensively mutating heavy and light CDR3 regions (Fig. 1F) in combination with high-throughput screening and antibody characterization assays.

We generated 305 single amino acid substitution variants of 2D3 (Fig. 1G), with the goal to only minimally disrupt the CD229 epitope recognized by 2D3, as well as to maintain its specificity and key CAR properties, including high CAR T cell expansion and lack of tonic signaling, which has been suggested to be more common in phage display-derived antibodies ${ }^{23}$.

\section{Single amino acid substitutions result in substantially reduced CD229 binding}

Identification of antibodies with reduced affinity represents a relatively uncommon objective in antibody discovery and poses unique challenges when developing appropriate screening approaches. For example, most common primary antibody screening assays, such as standard solid-phase binding assays using large sets of non-purified antibodies, are unable to differentiate between expression and affinity. As expected, this was also the case for 2D3, which showed a clear dependence of CD229 binding to antibody concentration, especially in concentration ranges commonly observed in standard high-throughput expression cultures (Fig. 2A). When screening for high-affinity antibodies this may be acceptable as the highest assay signals are likely the result of a combination of high affinity and high expression, both representing desirable properties. In the case of low affinity antibody screening, however, the potential conflation of low expressing/high affinity antibodies with high expressing/low affinity antibodies would render such data relatively meaningless. We therefore developed a highthroughput scFv quantification assay relying on the binding of Protein $L$ to the $\kappa$ light chain in 2D3 (Fig. 2B) and determined total scFv yields of all generated variants (Fig. 2C). As expected, substitutions to cysteine and proline generally resulted in poor antibody expression, while 
alanine and threonine appeared to be tolerated well in various positions (Fig. 2C). In addition, we found that some positions were able to accommodate almost any amino acid. This included some unexpected positions, such as asparagine in position $\mathrm{H} 5$, an exposed residue in the center of the groove formed by both CDR3 loops, as well as tryptophan in $\mathrm{H} 12$ and threonine in L9 (Fig. 2C). Importantly, several substitutions resulted in substantially improved expression, in line with established approaches to improve antibody stability via mutation of non-contact residues 24,25 . Following normalization of antibody concentrations, we next determined binding to recombinant CD229 in a standard solid-phase binding assay (Fig. 2D). While the majority of mutations did not reduce 2D3 variant binding to CD229, and some, as expected, increased binding, the comprehensive mutagenesis approach taken enabled the identification of a large set of binders showing various levels of reduced binding. While mutation of the outer residues in both CDR3s appeared to most drastically reduce binding, mutation of any position affected binding to some extent following individual amino acid substitutions. Importantly, alanine scanning ${ }^{26}$, one of the most widely used approaches to reduce protein-protein binding would not have resulted in a similarly comprehensive set of variants as alanine substitutions in many cases did not alter binding compared to parental 2D3 when other substitutions substantially affected binding. In addition, alanine substitutions never represented the variants with the lowest binding signal in any position.

Taken together, our data indicate that comprehensive single amino acid substitution may be preferable to conventional mutagenesis strategies and is able to generate large sets of antibodies showing reduced antigen binding even in already relatively low affinity binders. 
Based on scFv expression and CD229 binding data, we next selected 26 binders for downstream analyses.

Affinity tuning approach results in predominantly off-rate driven affinity reductions

To determine if single amino acid substitution mutagenesis in fact resulted in relevantly altered affinities and rate constants, we next purified (Suppl. Fig. 2) in vivo biotinylated 2D3 variant scFvs (Fig. 3A). We then subjected these antibodies to biolayer interferometry (BLI) using streptavidin biosensors and recombinant CD229 (Fig. 3B), allowing the use of relatively high analyte concentrations due to non-destructive sampling ${ }^{27}$, which facilitated characterization even of very weak binders (Fig. 3C). Affinities of 2D3 variants with single amino acid substitutions ranged from $175 \mathrm{nM}$ to $>10,000 \mathrm{nM}$ (Table 1 ) and we observed a very close correlation between antibody affinity $\left(K_{D}\right)$ and TRF binding (Suppl. Fig. 3). Differences in affinity were generally driven by faster off-rates (Table 1), which is likely related to the use of a solid phase binding assay for primary variant screening, which may have biased clone selection towards variants with reduced off-rates. In contrast to this finding, we observed a dramatic reduction in the on-rate of variants in which arginine in $\mathrm{H} 3$ was replaced (Fig. 3C, Table 1). A noted exception to this finding is RH3V, which in fact showed a faster on-rate but also a much faster off-rate (Fig. 3D). These data suggest a key role of arginine in the orchestration of the 2D3 epitope and among other possibilities might point to a model of 2D3 binding to CD229 in which $\mathrm{RH} 3$ binding facilitates interactions by other residues.

Single amino acid substitution together with BLI not only resulted in the identification of a set of clones with a wide range of mostly off-rate driven differences in affinity but also provided data regarding the mode of 2D3-CD229 binding, which may aide further lead optimization. 
Variant CAR T cells maintain anti-tumor activity and show improved selectivity, expansion and reduced trogocytosis

Optimal CAR affinity remains an active area of research but likely depends on various parameters, such as need for selectivity, epitope, as well as antigen- and CAR-density. Ideally, CAR affinity for a given target antigen will be chosen empirically, thus requiring a sufficiently large set of binders with different affinities available for CAR construction. We therefore converted all 26 antibodies into CAR constructs (Fig. 4A) and produced primary human CAR T cells using a standard manufacturing process (Fig. 4B). It has previously been shown that even parental 2D3-based CAR T cells can be manufactured without the CAR T cells targeting each other because CD229 is downregulated upon CD3/CD28-bead activation at the beginning of manufacturing ${ }^{21}$. However, it has been shown that certain CAR properties, such as tonic signaling, can result in vastly different expansion rates or even cause activation-induced cell death $^{28,29}$. We therefore first determined the viability and total CAR T cell yields on day 7 of manufacturing (Fig. 4C). While yields varied substantially between variant constructs, almost all showed expansion rates above wildtype 2D3-based CAR T cells. Considering the substantial differences in scFv expression (Fig. 2C), we next determined CAR surface expression of wildtype 2D3, all variant CAR constructs, as well as T cells expressing a CAR without a binding domain $(\Delta \mathrm{scFv})$. While all variant constructs expressed the linked GFP reporter, two constructs, FH10K and $\mathrm{AH} 1 \mathrm{~K}$, showed relatively low CAR surface expression and two other constructs, DH1OY and RH3N, did not show any CAR surface expression (Fig. 4D). One construct, TL9V, showed a bimodal distribution, potentially indicating recombination during retroviral packaging. We next determined whether mutagenesis had resulted in altered anti-tumor activity by determining 
killing of MM cells by all 2D3 variants at multiple relatively low effector-target ratios. We found that while several constructs indeed showed reduced tumor cell killing, likely due to the substantially reduced affinity of those variants, several constructs, including SH6A, GH4D, FH9Y, AH1I, AH1G, and FH9Q showed either equal or enhanced anti-tumor activity (Fig. 4E, Suppl. Fig. 4).

Next, we set out to determine whether reducing CAR affinity resulted in increased selectivity by measuring killing of purified T cells (Suppl. Fig. 5). At an effector-target ratio of 0.5:1, we found that, as expected, numerous constructs that had shown reduced cytotoxic activity against tumor cells, now also showed reduced killing of T cells (Fig. 4F). However, among some of the variants showing comparable or increased tumor cell killing at a low effector-target ratio, we were able to identify two constructs which showed dramatically reduced killing of T cells (Fig. 4F). The two candidates showing increased selectivity, FH9Q, and GH4D, in fact only showed T cell killing comparable to $\triangle \mathrm{scFv}$ control CAR T cells. CAR T cells based on variant FH9Q, which showed the lowest affinity while maintaining anti-tumor activity, as well as variant GH4D, which showed similar affinity but reduced CAR surface expression (Fig. 4D), spared healthy T cells even at a higher effector-target ratio and over a longer period of time (Fig. 4G), suggesting that FH9Q and GH4D CAR T cells are entirely unable to kill healthy T cells expressing physiologic levels of CD229. It had previously been shown that co-culture of 2D3-based CAR T cells resulted in the selection of a CD229low $\mathrm{T}$ cell population ${ }^{21}$. We observed the same phenomenon in this study for parental 2D3-based CAR T cells. However, co-culture with variant CAR T cells did not substantially change CD229 expression on healthy T cells (Fig. 4H). 
Other groups had shown that CAR T cells against various targets are able to strip antigen from tumor cells ${ }^{30}$ in a process called trogocytosis, resulting in antigen-negative tumor cells and potentially the targeting of CAR T cells by other CAR T cells after displaying the target antigen on their cell surface. Both are suspected to be potential tumor escape mechanisms in patients treated with CAR T cells. As it had previously been shown that trogocytosis correlates with receptor affinity ${ }^{31,32}$, we asked the question, whether single amino acid substitutions resulting in reduced affinity would also result in reduced trogocytosis. Indeed, we found that FH9Q- and GH4D-based CAR T cells showed a significantly reduced loss of CD229 from the tumor cells (Suppl. Fig. 6) as well as membrane transferred to the CAR T cells, indicating reduced levels of trogocytosis compared to 2D3 (Fig. 4I).

One of the two highly selective 2D3 variants, GH4D, is located adjacent to RH3, which appeared to be central to the 2D3-CD229 interaction. A docking simulation of wildtype 2D3 to the V2type domain of CD229 (Fig. 4J), which was previously shown to contain the 2D3 epitope ${ }^{21}$, indicates that $\mathrm{RH} 3$ and GH4 form a concave surface around CD229.S270 and CD229.Q271 with three hydrogen bonds between CD229.Q271 and RH3, and an additional bond between CD229.Q271 and GH4D. Disruptions of this motif via mutations containing relatively bulky sidechains, such as GH4D, may provide a structural basis for the reduced affinity observed in those variants. 


\section{DISCUSSION}

CAR T cells have revolutionized cancer immunotherapy, however, the development of safer and more effective CAR T cell approaches will be critical to their more widespread adoption and increased patient benefit. CAR affinity has recently come into focus due to increased tumor selectivity and improved CAR T cell function resulting from the use of low affinity CAR constructs $^{13-16}$. In addition, affinity tuning of CAR constructs opens the door to the targeting of new antigens that would otherwise be excluded due their potential toxicity profile. Here, we provide a systematic approach for the development of minimally modified low affinity antibody variants based on established and extensively tested antibodies that does not require detailed structural information including the antibody's exact epitope. We demonstrate that the variants produced using this approach indeed show significantly increased selectivity and improved CAR T cell function, while maintaining the original epitope and likely specificity. The majority of binders generated using our approach show a predominantly off-rate based reduction in affinity, likely as a consequence of using a solid phase binding assay for primary variant screening. While data regarding the relative contribution of on-rate and off-rate on CAR T cell signaling remain scarce, prior approaches to modulate CAR affinity have predominantly focused on changes in off-rates ${ }^{33-35}$. This includes one of the most advanced approaches already demonstrating the clinical benefits of the use of low affinity CAR constructs targeting CD19 ${ }^{13,34}$. Using the outlined approach, parental antibodies with very high affinities may require multiple iterations of mutagenesis to achieve a sufficient reduction in affinity and for some parental antibodies it may not be possible to generate variants within the required affinity window depending on their binding mode. However, we show that our affinity tuning approach is likely 
bioRxiv preprint doi: https://doi.org/10.1101/2021.12.06.471279; this version posted December 7, 2021. The copyright holder for this preprint (which was not certified by peer review) is the author/funder. All rights reserved. No reuse allowed without permission.

to produce a wide range of affinities and represents a robust workflow for the development of safer and more functional CAR T cells. 


\section{METHODS}

Cell lines and primary human cells

U266B1 and Phoenix-Ampho cells were purchased from ATCC and cultured according to ATCC instructions. Lenti-X 293T cells were purchased from Takara and cultured according to the manufacturer's instructions. Healthy donor buffy coats were obtained from the Blood Centers of America and the New York Blood Center and peripheral blood mononuclear cells were isolated from buffy coats by density gradient using FicollPaque (GE) as previously described ${ }^{21}$.

\section{Flow cytometry expression analysis}

Flow cytometry staining and analyses were performed as previously described ${ }^{21}$. CD229 surface expression was determined using a mouse monoclonal anti-CD229 antibody (clone: HLy9.1.25). Other antibodies used for flow cytometry analyses are listed in Supplementary Table 1. Commercially available antibodies were used at dilutions recommended by the respective manufacturer. For the analysis of CD229 expression on tumor cells and T cells from MM patients, data was acquired on a CytoFLEX LX (Beckman Coulter) and analyzed using Kaluza 2.1 (BC). All other flow cytometry data was acquired on an LSR Fortessa or LSR II flow cytometer (BD) and analyzed using FlowJo 10 (BD).

\section{Single amino acid substitution library production}

Parental 2D3 was cloned into pSANG10 ${ }^{36}$ and the single amino acid substitution library produced by was generated using high-throughput gene synthesis by Twist Bioscience. Individual mutations were confirmed by Sanger sequencing. To produce single-site biotinylated 
scFvs, a C-terminal AviTag was added to the pSANG10 expression constructs (pSANG10-Avi) and scFvs transformed into BL21[DE3] cells (Lucigen) containing the pBirAcm plasmid (Avidity).

scFv expression and purification

Monoclonal scFvs were expressed overnight in $25 \mathrm{~mL}$ MagicMedia E. coli autoexpression medium (Thermo-Fisher). Periplasmic extracts were generated from autoinduction cultures using standard procedures. For some experiments, scFvs were purified by metal affinity chromatography using NiNTA resin (Thermo). Concentrations of purified antibodies were determined by bicinchinonic assay (Thermo). pSANG10-Avi clones were expressed in the same way in the presence of $50 \mu \mathrm{M}$ free D-biotin (Sigma).

\section{IFN gamma ELISA}

Supernatants were harvested from 96 well overnight co-cultures and immediately frozen at -

$80^{\circ} \mathrm{C}$. IFN $\gamma$ concentrations were determined via standard curve using a commercial ELISA kit according to the manufacturer's instructions (Biolegend). Absorbance was measured on a multi-mode plate reader (Tecan).

\section{CAR T cell production}

Selected scFvs were cloned into a previously described second generation 4-1BB-based CAR construct $^{21}$ in the gammaretroviral SFG backbone. Amphotropic gammaretrovirus was generated by transfection of Phoenix-Ampho cells (ATCC \# CRL-3213) using the SFG-based transfer plasmids using Lipofectamine 2000 according to the manufacturer's instructions. Viruscontaining supernatants were concentrated with Retro-X concentrator (Takara). PBMCs were stimulated for 2 days with CD3/CD28 T cell activation beads (Thermo \# 11131D) in the presence 
of 40IU/mL IL2 (R\&D Systems \# 202-IL-010) in AIM V (Thermo) supplemented with 5\% human serum (Sigma \#H3667) and incubated at $37^{\circ} \mathrm{C} / 5 \% \mathrm{CO}_{2}$. Bead-stimulated cells were transferred to Retronectin-coated (Takara) virus-containing plates and incubated overnight. Transduction was repeated the next day before counting and diluting cells to $0.4 \times 10^{6}$ cells $/ \mathrm{ml}$. After the second transduction cells were grown for an additional 7 days before removing beads using a DynaMag-15 magnet (Thermo). IL-2 was replenished every 2 days to $40 \mathrm{IU} / \mathrm{mL}$. Cells were frozen in $90 \% \mathrm{FCS} / 10 \%$ DMSO and stored in liquid nitrogen.

\section{Trogocytosis assay}

CAR T cells were cocultured with target cells at the specified effector:target ratio. For some experiments, target cells were first labelled with membrane dyes PKH26 (Sigma \# MINI26) or BioTracker 555 (Sigma \# SCT107) according to the manufacturer's instructions. After the specified amount of time, cells were stained with antibodies and 500ng/mL 4',6-diamidine-2'phenylindole dihydrochloride (DAPI, Invitrogen \# D1306). Counting beads (Thermo \# PCB 100) were added after staining and cells were analyzed on a LSRFortessa or an LSR II flow cytometer (BD). Effector:target ratios and coculture times are listed in the figure legends.

\section{Flow cytometry-based cytotoxicity assay.}

A flow cytometry-based cytotoxicity assay was used to determine CAR T cell cytotoxicity against healthy T cells from the same healthy donor. T cells were collected using negative selection (Stemcell Technologies EasySep Human T Cell Isolation Kit) from autologous healthy donor PBMCs and stained with Cell Trace Far Red dye (CTD, Invitrogen) according to the mnufacturer's instructions. $5 \times 10^{4} \mathrm{~T}$ cells were co-cultured with different amounts of CAR T cells overnight in a 
round bottom 96 well plate at $37^{\circ} \mathrm{C} 5 \% \mathrm{CO} 2$. Following coculture, Accucheck counting beads (Life Technologies) and 500ng/mL DAPI were added to the cells. DAPI-CTD ${ }^{+}$T Cells were immediately quantified on an LSR II flow cytometer (BD).

Luciferase-based cytotoxicity assay.

To determine the cytotoxicity of variant CD229 CAR T cells against the multiple myeloma cell line U-266, U-266 cells were transduced with pHIV-Luc-ZsGreen lentivirus and sorted on a FACSaria flow cytometer (BD) for GFP expression. As with the flow cytometry-based cytotoxicity assay, $5 \times 10^{4}$ target $U-266$ cells were seeded in each well of a round bottom 96 well plate. Various ratios of CAR T cells were co-cultured with the $\mathrm{U}-266$ cells overnight at $37^{\circ} \mathrm{C} 5 \% \mathrm{CO}_{2}$. After the co culture, cells were suspended by gentle pipetting and $100 \mathrm{uL}$ were moved to a 96 well black flat bottom plate. $150 \mu \mathrm{g} / \mathrm{ml}$ D-luciferin (Gold Biotechnology Cat\# LUCNA-2G) was added to the cells and incubated for 5 mins at $37^{\circ} \mathrm{C}$. Luminescence was determined on a multimode plate reader (Tecan Spark).

\section{Time-resolved fluorescence assays.}

Two different time-resolved fluorescence (TRF) assays were used. To determine the concentration of scFv in the PPEs, 250ng of rat-anti-FLAG (clone: L5, Biolegend) in 50uL PBS was immobilized on a black 96-well plate (Greiner Bio-One) overnight at $4^{\circ} \mathrm{C}$. Plates were washed using an automated plate washer (Tecan HydroFlex) twice with PBS containing 0.1\% Tween-20, and twice with PBS, in between each incubation. After immobilization, all other incubations were performed at room temperature at 400rpm. Following immobilization, plates were blocked in 3\% non-fat milk in PBS (M-PBS). Then PPEs or purified 2D3 were added to plates in 
3\% M-PBS and incubated for $1 \mathrm{~h}$. Next, plates were incubated with $250 \mathrm{ng}$ biotinylated protein $\mathrm{L}$

(Thermo Scientific) in 3\% M-PBS for 1 h. Finally, plates were incubated with streptavidin-

Europium (PerkinElmer) in PBS for 30min. After a final wash, plates were incubated with DELFIA

Enhancement solution (PerkinElmer) for 10min. Time-resolved fluorescence was determined on

a multi-mode plate reader (Tecan Spark). A purified parental 2D3 standard was used to

calculate the scFv concentration in each PPE.

To determine the relative binding of variant scFvs to CD229, $5 \mu \mathrm{g} / \mathrm{ml}$ recombinant human

CD229 (R\&D Systems) was immobilized on a black 96-well plate (Greiner Bio-One) overnight at

$4^{\circ} \mathrm{C}$. After immobilization, plates were washed between each step and incubated at room

temperature at 400rpm as described above. Following immobilization, plates were blocked in

3\% M-PBS. Then, plates were incubated with 100 ng of each scFv in 3\% M-PBS. Next, plates

were incubated with anti-FLAG M2 (Sigma-Aldrich) in 3\% M-PBS. Finally, plates were incubated

with anti-mouse IgG-Europium antibody (PerkinElmer) for 1 hour. After a final wash, plates

were incubated with DELFIA Enhancement solution (PerkinElmer) for 10min. Time-resolved

fluorescence was determined on a multi-mode plate reader (Tecan Spark).

Biolayer Interferometry (BLI)

Streptavidin (SA) biosensensors (FortéBio) were hydrated in $1 \times$ Octet $^{\circledR}$ Kinetics Buffer

(Sartorius) for at least 10min. SA biosensors were loaded using the Octet K2 (Sartorius). A

baseline in Octet Kinetics Buffer was collected for $1 \mathrm{~min}$. Then the sensors were loaded with

variant scFvs using a threshold of $2 \mathrm{~nm}$ and subsequently blocked in Biocytin. Once loaded, sensors were placed back into the sensor tray and kept hydrated in kinetics buffer. Once

sensors were loaded, a kinetic run was performed. SA biosensors loaded with $2 \mathrm{~nm}$ of 
biotinylated scFv and blocked with biocytin went through a 60s baseline read in kinetics buffer, 50s association in 2, 1, 0.5, and 0.25 $\mu \mathrm{M}$ recombinant human CD229 (R\&D Systems) in kinetics buffer, and finally 60 s dissociation in kinetics buffer. BLI was run at $30^{\circ} \mathrm{C}$ and $10,000 \mathrm{rpm}$. Data was analyzed using Octet ${ }^{\circledast}$ K2 System Data Analysis 9.0 software.

\section{Structure and docking prediction}

Structures of the wildtype 2D3 scFv and the extracellular domain of CD229 were generated using AlphaFold ${ }^{37}$. The docking prediction was generated by HADDOCK $2.4^{38}$ by defining all 2D3 CDRs as active residues and the CD229 V-type 2 domain as passive residues. Structures were visualized using UCSF ChimeraX.

\section{Statistical Analysis}

Significance of differences in cell numbers, cytokine levels, and mean fluorescence intensity levels were calculated by Student's t-test. All statistical tests were performed using Prism 7 (GraphPad Software). Results were considered significant when $p<0.05$.

\section{Study approval}

The retrospective analysis of patient samples for the expression of CD229 was approved by the Institutional Review Board at the University of Utah (protocol 77285-19). 


\section{AUTHOR CONTRIBUTIONS}

EVM, and TL conceived the project, planned and performed experiments, analyzed data, and wrote the manuscript. JMB performed variant ScFv expression and CAR T cell production. PD and DPN performed flow cytometry analysis of MM samples. SVR, DN, JM, MS, MLO, and CL analyzed data. DA conceived the project and analyzed data. All authors reviewed and approved the manuscript.

\section{ACKNOWLEDGEMENTS}

This project was supported by a NCCN Young Investigator Award (T.L.), Huntsman Cancer Institute Experimental Therapeutics program supported by the National Cancer Institute of the National Institutes of Health under Award number P30CA042014. (D.A. and T.L.). We thank the Huntsman Cancer Institute in Salt Lake City, UT for the use of the Preclinical Research Resource (PRR), which performed the MM xenograft model, the ARUP Institute for Clinical and Experimental Pathology, and the University of Utah Flow Cytometry core facility, which assisted with flow cytometry analyses and cell sorting. pHIV-Luc-ZsGreen was a gift from Bryan Welm (Addgene \#39196), SFG.CNb30_opt.IRES.eGFP was a gift from Martin Pule (Addgene \# 22493), pSANG10-3F (Addgene plasmid \# 39264) was a gift from John McCafferty.

\section{CONFLICTS OF INTEREST}

ERV, TL, and DA are inventors on provisional patent application 63/285843 describing low-affinity CD229 antibodies and CAR T cells. SVR, DA, and TL are inventors on PCT application US2017/42840 "Antibodies and CAR T Cells for the Treatment of Multiple 
bioRxiv preprint doi: https://doi.org/10.1101/2021.12.06.471279; this version posted December 7, 2021. The copyright holder for this preprint (which was not certified by peer review) is the author/funder. All rights reserved. No reuse allowed without permission.

Myeloma" describing the therapeutic use of CD229 CAR T cells for the treatment of multiple myeloma. 


\section{REFERENCES}

1. Sadelain, M. CD19 CAR T Cells. Cell 171, 1471 (2017).

2. Raje, N., et al. Anti-BCMA CAR T-Cell Therapy bb2121 in Relapsed or Refractory Multiple Myeloma. The New England journal of medicine 380, 1726-1737 (2019).

3. Mullard, A. FDA approves 100th monoclonal antibody product. Nat Rev Drug Discov (2021).

4. Cheever, M.A., et al. The prioritization of cancer antigens: a national cancer institute pilot project for the acceleration of translational research. Clin Cancer Res 15, 53235337 (2009).

5. Sun, S., Hao, H., Yang, G., Zhang, Y. \& Fu, Y. Immunotherapy with CAR-Modified T Cells: Toxicities and Overcoming Strategies. Journal of immunology research 2018, 23861872386187 (2018).

6. Hirayama, A.V. \& Turtle, C.J. Toxicities of CD19 CAR-T cell immunotherapy. American Journal of Hematology 94, S42-S49 (2019).

7. Mamonkin, M., Rouce, R.H., Tashiro, H. \& Brenner, M.K. A T-cell-directed chimeric antigen receptor for the selective treatment of T-cell malignancies. Blood 126, 983-992 (2015).

8. Liu, X., Zhang, N. \& Shi, H. Driving better and safer HER2-specific CARs for cancer therapy. Oncotarget 8, 62730-62741 (2017).

9. Morgan, R.A., et al. Case Report of a Serious Adverse Event Following the Administration of T Cells Transduced With a Chimeric Antigen Receptor Recognizing ERBB2. Molecular Therapy 18, 843-851 (2010). 
10. Chmielewski, M., Hombach, A., Heuser, C., Adams, G.P. \& Abken, H. T Cell Activation by Antibody-Like Immunoreceptors: Increase in Affinity of the Single-Chain Fragment Domain above Threshold Does Not Increase T Cell Activation against Antigen-Positive Target Cells but Decreases Selectivity. The Journal of Immunology 173, 7647 (2004).

11. Liu, X., et al. Affinity-Tuned ErbB2 or EGFR Chimeric Antigen Receptor T Cells Exhibit an Increased Therapeutic Index against Tumors in Mice. Cancer Res 75, 3596-3607 (2015).

12. Turatti, F., et al. Redirected activity of human antitumor chimeric immune receptors is governed by antigen and receptor expression levels and affinity of interaction. $J$ Immunother 30, 684-693 (2007).

13. Ghorashian, S., et al. Enhanced CAR T cell expansion and prolonged persistence in pediatric patients with ALL treated with a low-affinity CD19 CAR. Nature Medicine 25, 1408-1414 (2019).

14. Heitner, T., et al. Selection of cell binding and internalizing epidermal growth factor receptor antibodies from a phage display library. J Immunol Methods 248, 17-30 (2001).

15. Yang, J., et al. Therapeutic potential and challenges of targeting receptor tyrosine kinase ROR1 with monoclonal antibodies in B-cell malignancies. PloS one 6, e21018-e21018 (2011).

16. Hudecek, M., et al. Receptor Affinity and Extracellular Domain Modifications Affect Tumor Recognition by ROR1-Specific Chimeric Antigen Receptor T Cells. Clinical Cancer Research 19, 3153 (2013).

17. Ali, S.A., et al. T cells expressing an anti-B-cell maturation antigen chimeric antigen receptor cause remissions of multiple myeloma. Blood 128, 1688-1700 (2016). 
18. Friedman, K.M., et al. Effective Targeting of Multiple B-Cell Maturation AntigenExpressing Hematological Malignances by Anti-B-Cell Maturation Antigen Chimeric Antigen Receptor T Cells. Hum Gene Ther 29, 585-601 (2018).

19. Garfall, A.L., et al. Chimeric Antigen Receptor T Cells against CD19 for Multiple Myeloma. The New England journal of medicine 373, 1040-1047 (2015).

20. Boucher, K., et al. Stemness of B-cell progenitors in multiple myeloma bone marrow. Clin Cancer Res 18, 6155-6168 (2012).

21. Radhakrishnan, S.V., et al. CD229 CAR T cells eliminate multiple myeloma and tumor propagating cells without fratricide. Nat Commun 11, 798 (2020).

22. Nicholson, I.C., et al. Construction and characterisation of a functional CD19 specific single chain Fv fragment for immunotherapy of B lineage leukaemia and lymphoma. Mol Immunol 34, 1157-1165 (1997).

23. Spencer, S., Bethea, D., Raju, T.S., Giles-Komar, J. \& Feng, Y. Solubility evaluation of murine hybridoma antibodies. MAbs 4, 319-325 (2012).

24. Tiller, T., et al. A fully synthetic human Fab antibody library based on fixed VH/VL framework pairings with favorable biophysical properties. MAbs 5, 445-470 (2013).

25. Wu, S.J., et al. Structure-based engineering of a monoclonal antibody for improved solubility. Protein Eng Des Sel 23, 643-651 (2010).

26. Weiss, G.A., Watanabe, C.K., Zhong, A., Goddard, A. \& Sidhu, S.S. Rapid mapping of protein functional epitopes by combinatorial alanine scanning. Proc Natl Acad Sci U S A 97, 8950-8954 (2000). 
27. Petersen, R.L. Strategies Using Bio-Layer Interferometry Biosensor Technology for Vaccine Research and Development. Biosensors (Basel) 7(2017).

28. Long, A.H., et al. 4-1BB costimulation ameliorates T cell exhaustion induced by tonic signaling of chimeric antigen receptors. Nat Med 21, 581-590 (2015).

29. Lynn, R.C., et al. c-Jun overexpression in CAR T cells induces exhaustion resistance. Nature 576, 293-300 (2019).

30. Hamieh, M., et al. CAR T cell trogocytosis and cooperative killing regulate tumour antigen escape. Nature 568, 112-116 (2019).

31. Richardson, S.I., et al. IgG3 enhances neutralization potency and Fc effector function of an HIV V2-specific broadly neutralizing antibody. PLoS Pathog 15, e1008064 (2019).

32. Chung, B., et al. Antigen-specific inhibition of high-avidity T cell target lysis by lowavidity T cells via trogocytosis. Cell Rep 8, 871-882 (2014).

33. Arcangeli, S., et al. Balance of Anti-CD123 Chimeric Antigen Receptor Binding Affinity and Density for the Targeting of Acute Myeloid Leukemia. Mol Ther 25, 1933-1945 (2017).

34. Roddie, C., et al. Durable Responses and Low Toxicity After Fast Off-Rate CD19 Chimeric Antigen Receptor-T Therapy in Adults With Relapsed or Refractory B-Cell Acute Lymphoblastic Leukemia. J Clin Oncol, JCO2100917 (2021).

35. Drent, E., et al. A Rational Strategy for Reducing On-Target Off-Tumor Effects of CD38Chimeric Antigen Receptors by Affinity Optimization. Mol Ther 25, 1946-1958 (2017).

36. Schofield, D.J., et al. Application of phage display to high throughput antibody generation and characterization. Genome biology 8, R254 (2007). 
37. Jumper, J., et al. Highly accurate protein structure prediction with AlphaFold. Nature 596, 583-589 (2021).

38. van Zundert, G.C.P., et al. The HADDOCK2.2 Web Server: User-Friendly Integrative Modeling of Biomolecular Complexes. J Mol Biol 428, 720-725 (2016). 


\section{FIGURES}
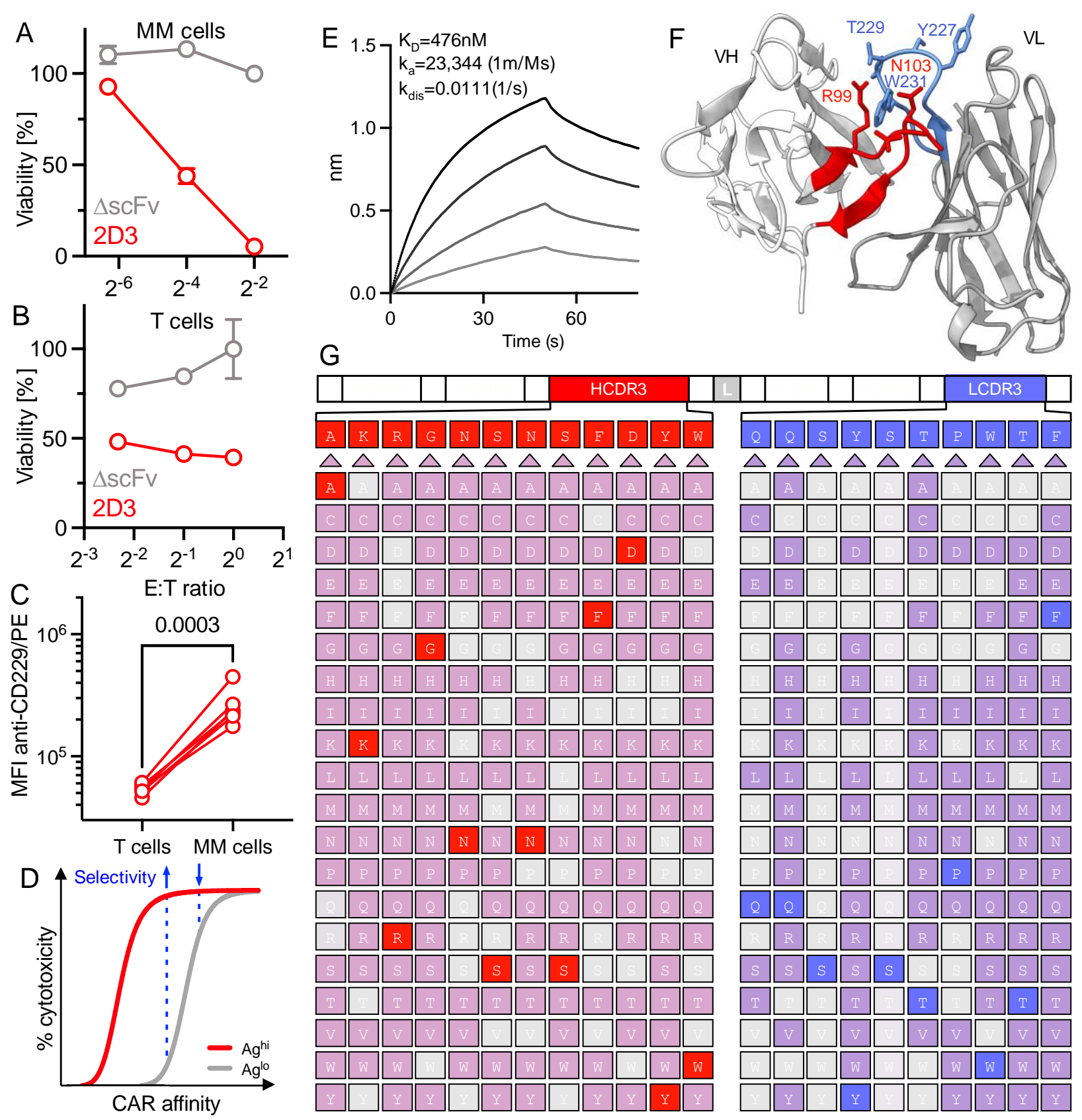

Figure 1: Generation of 2D3-based CDR3 variant library for the generation of low affinity

CD229 antibodies with increased selectivity. (A) Killing of CD229+ MM cell line U266B1

expressing luciferase by CD229 CAR T cells (2D3) or T cells expressing a CAR without a binding domain $(\Delta \mathrm{scFv})$ as determined by luminescence assay. Data indicate mean \pm S.D. from technical 
replicates ( $N=3$ ). (B) Killing of healthy human T cells by CD229 CAR T cells (2D3) as determined by flow cytometry cytotoxicity assay. Data indicate mean \pm S.D. from technical replicates $(N=3)$. (C) Surface expression of CD229 on MM and T cells from patients with relapsed/refractory MM $(N=6)$. Expression was determined after staining with an anti-CD229 antibody (clone: HLy9.1.25) on a CytoFLEX LX flow cytometer (Beckman Coulter). (D) Schema of relationship between CAR affinity and targeting of cells expressing high antigen levels ( $\left.\mathrm{gg}^{\text {hi }}\right)$ and low antigen levels $\left(\mathrm{Ag}^{\mathrm{lo}}\right)$. (E) Sensorgram of 2D3 binding to CD229. Equilibrium and rate constants of the 2D3 scFv were determined by biolayer interferometry (BLI). Biotinylated 2D3 was immobilized on a streptavidin biosensor and the recombinant extracellular domain of CD229 was added in the following concentrations: $2 \mu \mathrm{M}, 1 \mu \mathrm{M}, 0.5 \mu \mathrm{M}, 0.25 \mu \mathrm{M}$. Sensorgram indicates binding curves for descending CD229 concentrations. Plot shows a representative result of three independent experiments. (F) Structure of the 2D3 scFv as determined by AlphaFold2 with the GS-linker omitted. CDR3 loops of the variable heavy (red) and the variable light (blue) chains as well as exposed residues are highlighted. (G) Schema of single amino acid substitution 2D3 CDR3 variant library indicating represented residues. Missing variants (grey) and wild-type residues (red/blue) are highlighted. 

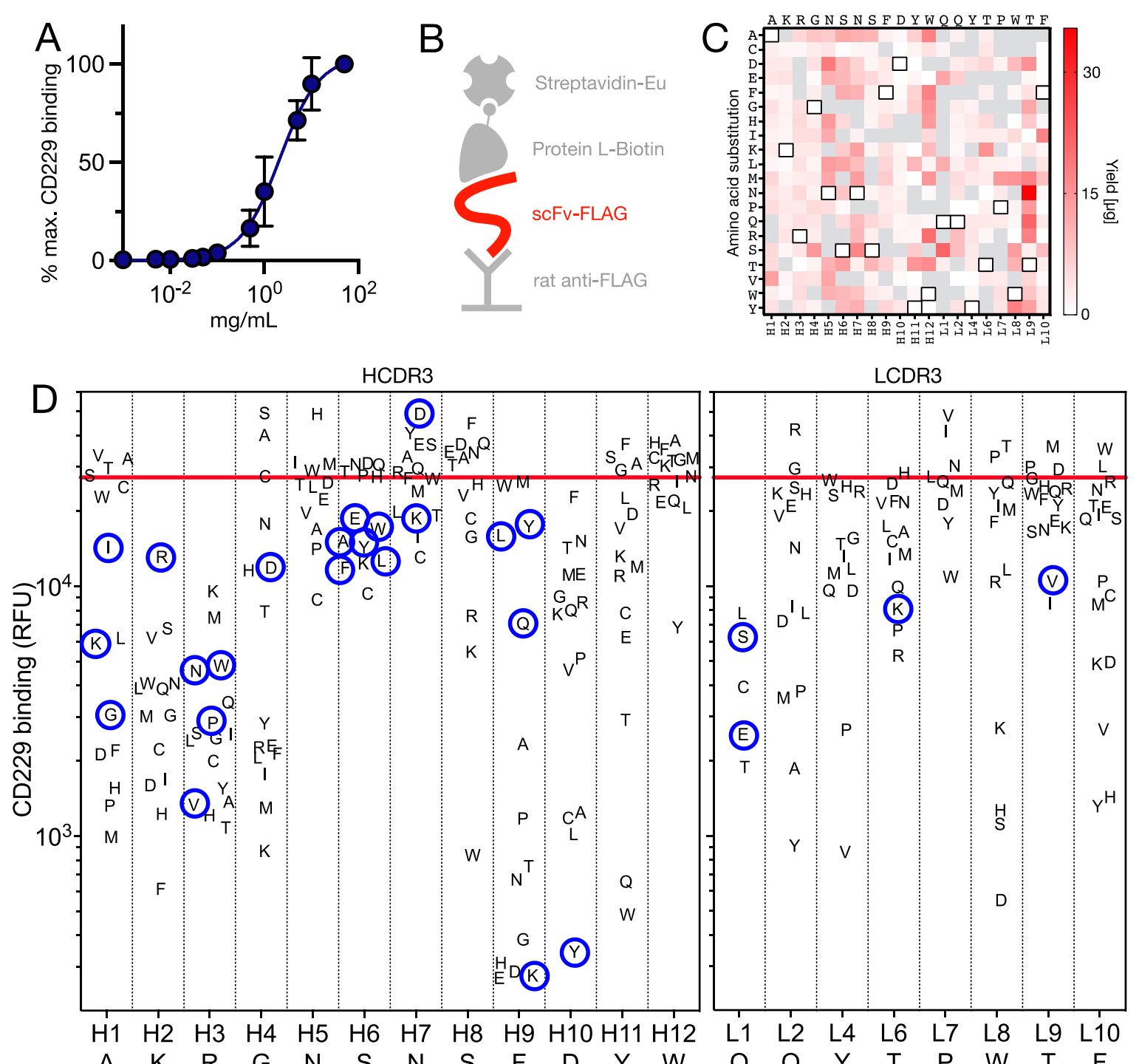

Figure 2: Single amino acid substitutions result in substantially reduced CD229 binding. (A)

Concentration-dependence of 2D3 scFv binding to recombinant CD229 as determined by solidphase time-resolved fluorescence assay. Data indicate mean \pm S.D. from technical replicates $(N=3)$. (B) Schema of solid-phase assay for the determination of FLAG-tagged scFv concentrations using rat $\lg 2 \mathrm{a} \lambda$ anti-FLAG antibody, biotinylated Protein $\mathrm{L}$, and streptavidinEu. (C) Total yield of 2D3 variants from $25 \mathrm{~mL}$ autoinduction cultures as determined by assay illustrated in 2B. White squares with black outlines indicate amino acids in wildtype 2D3. Plot 
shows representative result of two independent experiments. (D) Binding of all expressed 2D3 variants at $2 \mathrm{ng} / \mu \mathrm{l}$ to recombinant CD229 as determined by solid-phase time-resolved fluorescence assay. Red line indicates wildtype 2D3 binding to CD229. Blue circles indicate variants selected for downstream assays based on amino acid position and binding signal. Plot shows representative result of two independent experiments. 


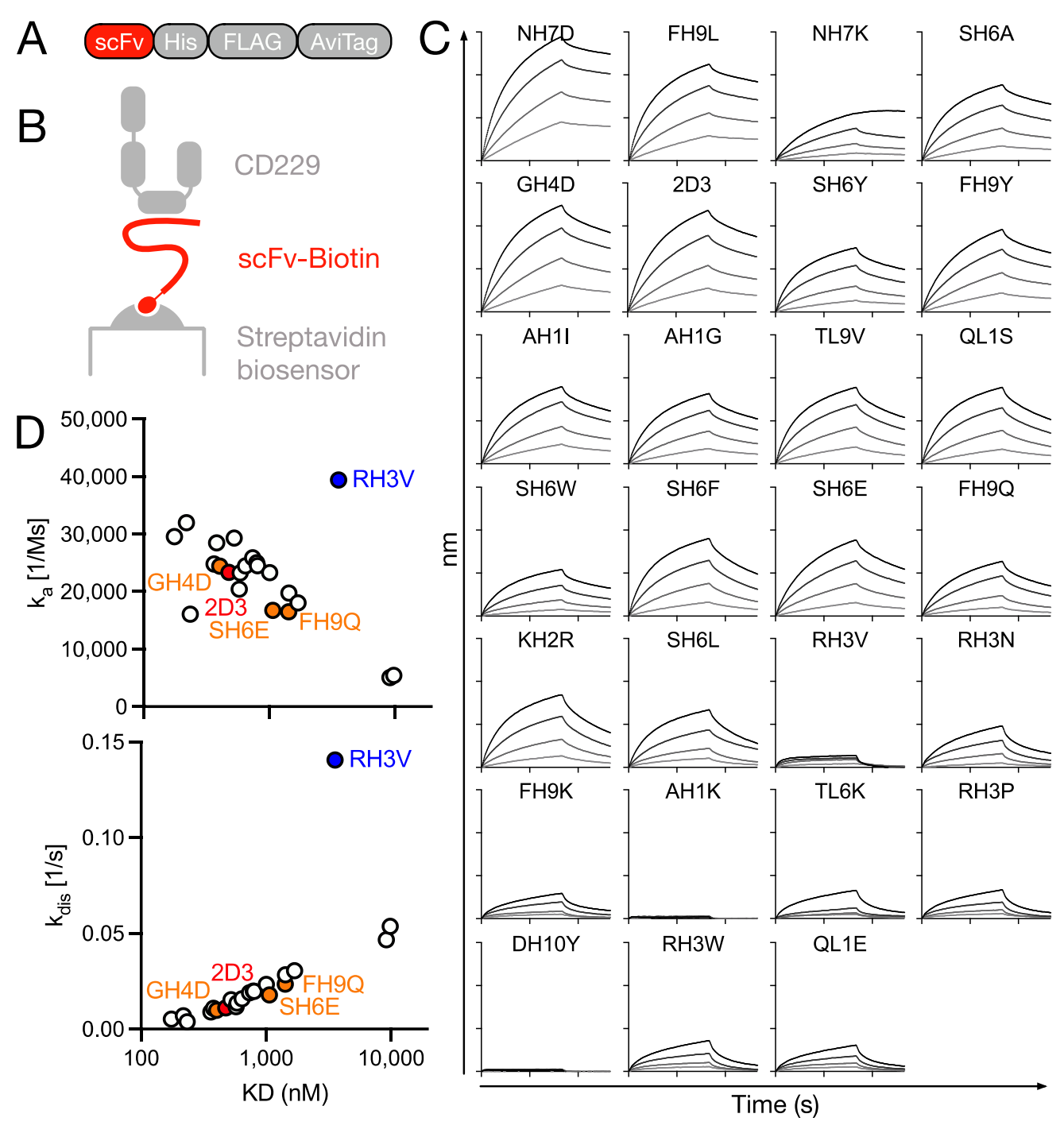

Figure 3: Affinity tuning approach results in predominantly off-rate-driven affinity reductions.

(A) Schema of construct used for production of biotinylated 2D3 scFv variants including C-

terminal Avitag to facilitate in vivo biotinylation. (B) Schema of biolayer interferometry (BLI) setup used for kinetic characterization of CD229 binding. Biotinylated 2D3 variants were immobilized on streptavidin biosensors and the recombinant extracellular domain of CD229 was added in the following concentrations: $2 \mu \mathrm{M}, 1 \mu \mathrm{M}, 0.5 \mu \mathrm{M}, 0.25 \mu \mathrm{M}$. (C) Sensorgrams of 2D3 variants were determined using an Octet K2 (Sartorius). Plots show representative result of two 
bioRxiv preprint doi: https://doi.org/10.1101/2021.12.06.471279; this version posted December 7, 2021. The copyright holder for this preprint (which was not certified by peer review) is the author/funder. All rights reserved. No reuse allowed without permission.

independent experiments. (D) Correlation between rate and equilibrium constants of 2D3 variant scFvs as determined by BLI. 

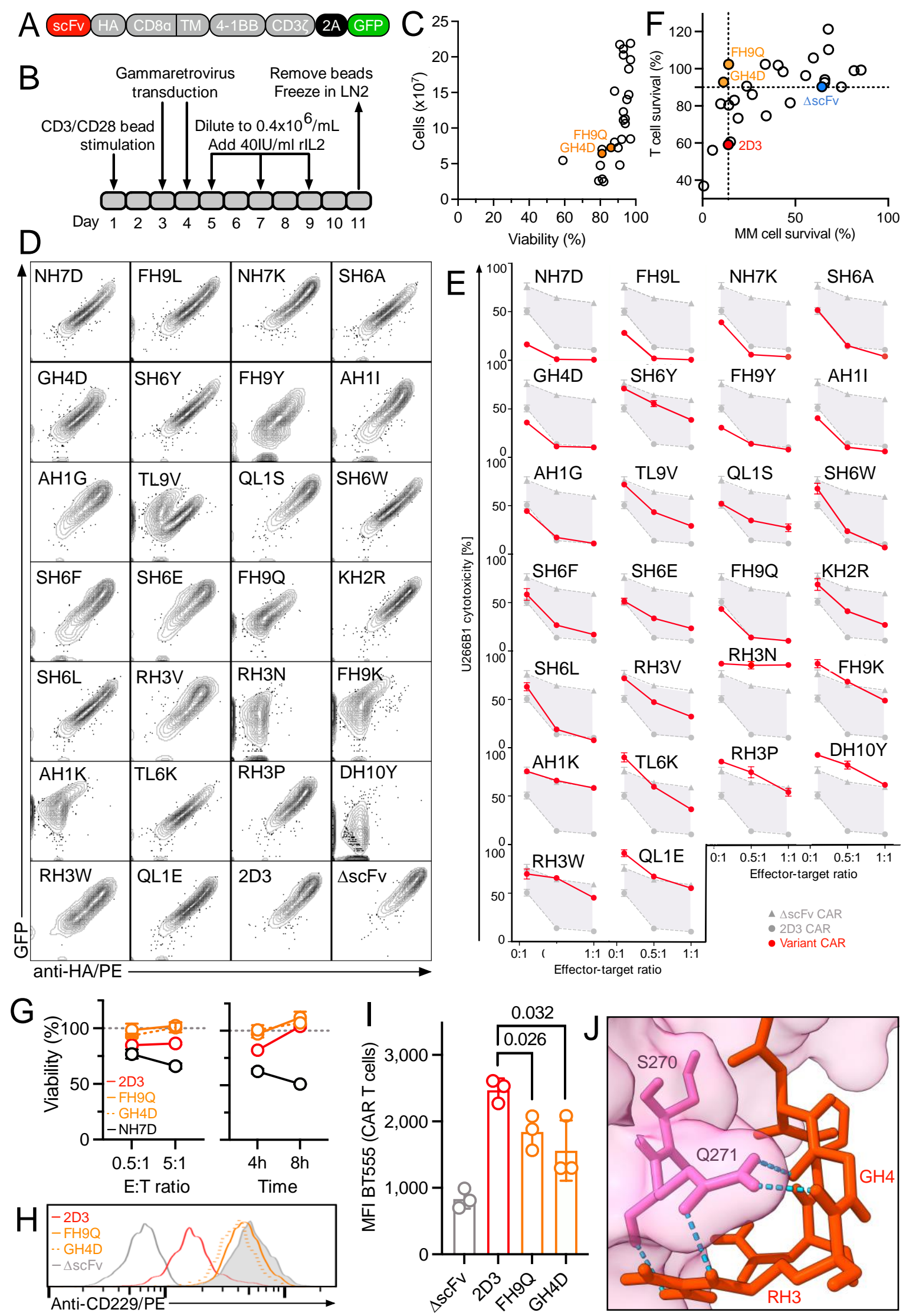
Figure 4: Selective HCDR3 variants maintain anti-tumor activity but exhibit minimal T cell killing over time and reduced trogocytosis. (A) Schema of 4-1BB-based second-generation CAR construct with GFP reporter. (B) Schema of gammaretrovirus-based CAR T cell production process. (C) Correlation between CAR T cell yield and vaibility of 26 2D3 variant CARs. (D) Surface expression of 2D3 variant CARs and GFP reporter expression as determined by anti-HA staining using flow cytometry. (E) Cytotoxic activity of 2D3 variant CAR T cells against MM cell line U266B1 expressing luciferase at different effector-target ratios using a luminescence-based cytotoxicity assay. Data indicate mean \pm S.D. from technical replicates $(N=3)$. (F) Correlation of cytotoxic activity of 2D3 variant CAR T cells against MM cells and T cells overnight at an effector-target ratio of 0.5:1. Data indicate mean killing (fold of wildtype 2D3) \pm S.D. from technical replicates $(N=3)$. Candidates were selected for downstream assays based on increased selectivity and anti-tumor activity (orange). (G) T cell killing by 2D3 and FH9Q variant CAR T cells at different effector-target ratios and for different durations as determined by flow cytometry. Data indicate mean \pm S.D. from technical replicates $(N=3)$. (H) Surface expression of CD229 on healthy T cells after co-culture with indicated CAR T cells for 8h at an effector target ratio of 5:1 as determined by flow cytometry. (I) Membrane transfer from U266B1 cells to variant CAR T cells after $4 \mathrm{~h}$ co-culture at an effector-target ratio of 4:1 as determined following Biotracker 555 staining of U266B1 cells using flow cytometry. Data indicate mean \pm S.D. from technical replicates $(N=3)$. Statistical differences between conditions were determined by two-sided Student's t test. (J) Docking structure of human CD229 (pink) with wildtype 2D3 (red) as predicted by HADDOCK2.4 defining residues within the CD229 V2-type domain as passive and 
bioRxiv preprint doi: https://doi.org/10.1101/2021.12.06.471279; this version posted December 7, 2021. The copyright holder for this preprint (which was not certified by peer review) is the author/funder. All rights reserved. No reuse allowed without permission.

HCDR3 residues showing reduced binding following mutation (Fig. 2D) as active. Key residues are annotated according to either full-length CD229 or HCDR3 position. 


\section{TABLES}

\begin{tabular}{lrrr} 
Clone & KD (nM) & ka (1/M.s) & kdis (1/s) \\
\hline NH7D & 175 & 29590 & 0.0052 \\
\hline FH9L & 218 & 32007 & 0.0070 \\
\hline NH7K & 234 & 16084 & 0.0038 \\
\hline SH6A & 380 & 28502 & 0.0108 \\
\hline GH4D & 402 & 24421 & 0.0098 \\
\hline 2D3 & 476 & 23344 & 0.0111 \\
\hline SH6Y & 524 & 29323 & 0.0154 \\
\hline FH9Y & 575 & 20420 & 0.0117 \\
\hline AH1I & 585 & 23268 & 0.0136 \\
\hline AH1G & 644 & 24487 & 0.0158 \\
\hline TL9V & 736 & 25885 & 0.0190 \\
\hline QL1S & 791 & 25039 & 0.0198 \\
\hline SH6W & 803 & 24513 & 0.0197 \\
\hline SH6F & 1,006 & 23307 & 0.0234 \\
\hline SH6E & 1,065 & 16737 & 0.0178 \\
\hline FH9Q & 1,425 & 16507 & 0.0235 \\
\hline KH2R & 1,431 & 19747 & 0.0283 \\
\hline SH6L & 1,689 & 18046 & 0.0305 \\
\hline RH3V & 3,565 & 39427 & 0.1406 \\
\hline RH3N & 9,157 & 5088 & 0.0466 \\
\hline FH9K & 9,835 & 5452 & 0.0536 \\
\hline AH1K & $>10,000$ & 50315 & 0.9477 \\
\hline TL6K & $>10,000$ & 1337 & 0.0635 \\
\hline RH3P & $>10,000$ & 755 & 0.0679 \\
\hline DH10Y & $>10,000$ & 8164 & 0.8991 \\
\hline RH3W & $>10,000$ & 595 & 0.0703 \\
\hline QL1E & $>10,000$ & 558 & 0.0688
\end{tabular}

Table 1: Single amino acid substitution results in broad range of affinities. Equilibrium and rate constants of 2D3 variants were determined using an Octet K2 (Sartorius). Data are representative of two independent experiments. Parental 2D3 is shown in red and variants with increased selectivity are shown in orange. 